\title{
Kinetic Monte Carlo simulations of the growth of polymer crystals
}

\author{
Jonathan P. K. Doye ${ }^{\mathrm{a})}$ and Daan Frenkel \\ FOM Institute for Atomic and Molecular Physics, Kruislaan 407, 1098 SJ Amsterdam, The Netherlands
}

(Received 5 August 1998; accepted 26 October 1998)

\begin{abstract}
Based upon kinetic Monte Carlo simulations of crystallization in a simple polymer model we present a new picture of the mechanism by which the thickness of lamellar polymer crystals is constrained to a value close to the minimum thermodynamically stable thickness, $l_{\min }$. The free energetic costs of the polymer extending beyond the edges of the previous crystalline layer and of a stem being shorter than $l_{\min }$ provide upper and lower constraints on the length of stems in a new layer. Their combined effect is to cause the crystal thickness to converge dynamically to a value close to $l_{\min }$ where growth with constant thickness then occurs. This description contrasts with those given by the two dominant theoretical approaches. However, at small supercoolings the rounding of the crystal profile does inhibit growth as suggested in Sadler and Gilmer's entropic barrier model. (C) 1999 American Institute of Physics. [S0021-9606(99)50305-X]
\end{abstract}

\section{INTRODUCTION}

Upon crystallization from solution and the melt many polymers form lamellae where the polymer chain traverses the thin dimension of the crystal many times folding back on itself at each surface. ${ }^{1}$ Although lamellar crystals were first observed over 40 years ago their physical origin is still controversial. It is agreed that the kinetics of crystallization are crucial since extended-chain crystals are thermodynamically more stable than lamellae. However, the explanations for the dependence of the lamellar thickness on temperature offered by the two dominant theoretical approaches appear irreconcilable. ${ }^{2,3}$ The lamellar thickness is always slightly greater than $l_{\min }$, the minimum thickness for which the crystal is thermodynamically more stable than the melt; $l_{\min }$ is approximately inversely proportional to the degree of supercooling. ${ }^{4}$

The first theory, which was formulated by Lauritzen and Hoffman (LH) soon after the initial discovery of the chainfolded crystals, ${ }^{5-7}$ invokes surface nucleation of a new layer on the thin side faces of the lamellae as the key process. It assumes that there is an ensemble of crystals of different thickness, each of which grows with constant thickness. The crystals which grow most rapidly dominate this ensemble, and so the average value of the thickness in the ensemble, which is equated with the observed thickness, is slightly larger than the thickness for which the crystals have the maximum growth rate. The growth rates are derived by assuming that a new crystalline layer grows by the deposition of a succession of stems (straight portions of the polymer that traverse the crystal once) along the growth face. The two main factors that determine the growth rate are the thermodynamic driving force and the free energy barrier to deposition of the first stem in a layer. The former only favors crystallization when the thickness is greater than $l_{\min }$; the latter increases with the thickness of the crystal because of the free

\footnotetext{
a) Present address: University Chemical Laboratory, Lensfield Road, Cambridge CB2 1EW, UK; electronic mail: jon@ clust.ch.cam.ac.uk
}

energetic cost of creating the two new lateral surfaces on either side of the stem and makes crystallization of thick crystals prohibitively slow. Therefore, the growth rate passes through a maximum at an intermediate value of the thickness which is slightly greater than $l_{\min }$.

The second approach, which was developed by Sadler and Gilmer and has been termed the entropic barrier model, is based upon the interpretation of kinetic Monte Carlo simulations $^{8,9}$ and rate-theory calculations ${ }^{10-12}$ of a simplified model of polymer crystal growth. As with the surface nucleation approach, the observed thickness is suggested to result from the competition between a driving force and a free energy barrier contribution to the growth rate. However, a different cause for the free energy barrier is postulated. As the polymer surface in the model can be rough, it is concluded that details of surface nucleation of new layers are not important. Instead, the outer layer of the crystal is found to be thinner than that in the bulk; this rounded crystal profile prevents further crystallization. ${ }^{12}$ Therefore, growth of a new layer can only begin after a fluctuation to an entropically unlikely configuration in which the crystal profile is "squared-off." As this fluctuation becomes more unlikely with increasing crystal thickness, the entropic barrier to crystallization increases with thickness.

Although both approaches are able to describe some of the basic phenomenology of polymer crystallization correctly, both have questionable aspects. As Frank and Tosi pointed out, one implication of the LH assumptions is that the thickness of an individual crystal should not vary even when the temperature is changed. ${ }^{13}$ However, this is clearly contradicted by experiment: when the temperature is changed the thickness of a growing crystal adjusts to the new temperature producing a step on the lamella. ${ }^{14,15}$ Sadler has also extensively questioned the adequacy of the LH approach, in particular to explain curved crystal habits and the effect of twins on the growth rate. ${ }^{16-20}$ Furthermore, there is the so-called " $\delta l$ catastrophe:" at too large supercoolings the predicted thickness goes to infinity unless a parameter in 
the model, the apportionment factor $\Psi$, is rather arbitrarily set to zero. ${ }^{7}$

In the entropic barrier approach it is not clear whether approximations such as the implicit representation of the chain connectivity and chain folds by a set of simple growth rules and neglect of the energetic contribution of chain folds to the free energy of the fold surface compromise its conclusions. Furthermore, although the simulations have been interpreted in terms of an entropic barrier, no direct evidence of this free energy barrier has been provided. ${ }^{21}$

There have been a number of works which have taken the basic LH approach but relaxed some of the constraints. For example, both Frank and Tosi ${ }^{13}$ and Lauritzen and Passaglia $^{22}$ have allowed the stem lengths within a layer and between layers to vary. Later, Point allowed the first stem to be deposited in steps rather than as a complete stem; this change prevents the $\delta l$ catastrophe. ${ }^{23,24}$ In the same spirit DiMarzio and Guttman wanted to allow every stem to be deposited in steps and to allow a fold to be formed at any step; however they could only consider very restricted cases. ${ }^{25}$ The approach used in all these papers was to attempt to find analytical solutions to the rate-theory equations, but as the number of possible pathways considered increased the problems became increasingly intractable. The natural solution to this difficulty is to use computer simulation techniques such as kinetic Monte Carlo (KMC). However, all these papers are over 15 years old, and at that time such an approach was not so feasible; only Point made an attempt to apply computational methods to the problem. ${ }^{26}$

Therefore, a thorough application of computer simulation to an unrestricted version of the LH approach is long overdue. In this article we do just this. We examine the growth of new crystalline layers from solution on a surface that represents the growth face of a polymer crystal when there are no constraints on the stem length or on when a fold can form. Our model is, in some ways, midway between the original LH model and the model used by Sadler and Gilmer. In the latter, a realistic representation of the polymer is sacrificed in order to be able to consider, for example, rough growth surfaces. Consequently, our work, as well as providing a test of the two theories, could play an important role in understanding the relationship between them. This work should also be seen in the context of an increasing application of simulation to study processes relevant to polymer crystallization. ${ }^{27-31} \mathrm{~A}$ brief presentation of some of the results in this article has appeared elsewhere. ${ }^{32}$

\section{METHODS}

In our simulations the polymer is represented by a selfavoiding walk on a simple cubic lattice. There is an attractive energy, $-\epsilon$, between nonbonded polymer units on adjacent lattice sites and an energetic penalty, $\epsilon_{g}$, for each kink (a "gauche" bond) in the chain. As the surface in our system represents the growth face of a polymer crystal, the $-\epsilon$ interaction also applies to polymer units in contact with the surface. $\epsilon$ can be considered to be an effective interaction representing the combined effects of polymer-polymer, polymer-solvent and solvent-solvent interactions, and so we can use our model in a simplified representation of the crys-

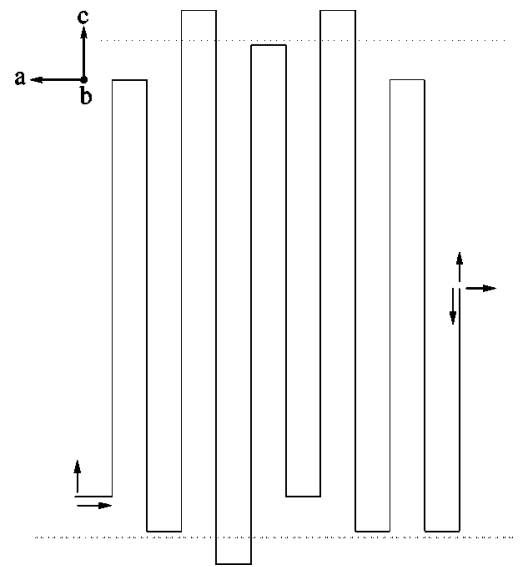

FIG. 1. An example of the configuration for the crystalline portion of the polymer which shows the possible moves (shown by arrows) in the kinetic Monte Carlo simulation. For this configuration, where growth is allowed to occur from either end, there are five possible moves. The dashed lines mark the boundaries of the underlying surface.

tallization of a semi-flexible polymer from solution. The behavior of the polymer is controlled by the ratio $k T / \epsilon$; large values can be considered as either high temperature or good solvent conditions, and low values as low temperature or bad solvent conditions. The parameter $\epsilon_{g}$ defines the stiffness of the chain. The polymer chain is flexible at $\epsilon_{g}=0$ and becomes stiffer as $\epsilon_{g}$ increases. Here, we use $\epsilon_{g}=8 \epsilon$, however similar results are obtained at any positive $\epsilon_{g} .{ }^{33}$

This interaction scheme has recently been used to investigate the phase behavior of isolated homopolymers ${ }^{34-36}$ and a homopolymer in the presence of a surface. ${ }^{37}$ The low temperature behavior is of particular interest since the polymers were found to adopt cuboidal ${ }^{36}$ (rectangular when on a surface ${ }^{37}$ ) "crystalline" configurations involving chain folding. However, these structures were observed for thermodynamic reasons - they are lowest in energy-whereas chain folding occurs in polymer crystallization for kinetic reasons.

In our simulations we wish to investigate the growth of new crystalline layers on the growth face of a crystal. To achieve this we choose not to use conventional Monte Carlo or molecular dynamics simulations since it is not feasible with these methods to probe the desired time and length scales. Instead we use kinetic Monte Carlo. ${ }^{38}$ However, this, of course, means that we have to make certain assumptions about the processes contributing to polymer crystallization. In our model when a polymer crystallizes on the growth face it forms a two-dimensional crystalline layer. The crystals formed only have adjacent re-entry of the stems; i.e., only tight folds where the new stem is adjacent to the previous stem are allowed. An example of such a configuration is shown in Fig. 1.

To make the problem more computationally tractable we only model the crystalline portion of the polymer explicitly. The rest of the chain is assumed to behave like an ideal coil. If we consider the coil to be a two-dimensional (2D) coil adsorbed onto the surface the free energy is

$$
A_{\text {ideal }, 2 \mathrm{D}}=-N_{\text {coil }} k T \log \left(1+2 \exp \left(-\beta \epsilon_{g}\right)\right)-N_{\text {coil }} \epsilon,
$$


whereas if the coil adopts a three-dimensional (3D) configuration in solution the free energy is

$$
A_{\text {ideal,3D }}=-N_{\text {coil }} k T \log \left(1+4 \exp \left(-\beta \epsilon_{g}\right)\right),
$$

where $N_{\text {coil }}$ is the number of units in the coil state. These expressions ignore any energetic contributions from contacts between polymer units in the coil. This approximation becomes worse as the temperature decreases, because the disordered chain would then be expected to have a dense collapsed conformation. ${ }^{36}$

For a single homopolymer (with the current interactions scheme and with all units explicitly represented) in the presence of an infinite polymer-like surface, the lowest energy configuration is a two-dimensional crystalline configuration. Simulations have shown that upon melting of the crystalline polymer a two-dimensional disordered state was formed for all values of $\epsilon_{g}$, and only at higher temperature does the polymer become more three dimensional. ${ }^{37}$ This suggests that Eq. (1) is an appropriate form by which to describe the coil. However, when considering growth on a thin lamella, the surface area on which the coil can absorb is greatly reduced. To stay on the surface the coil must adopt an anisotropic configuration in the plane which would reduce its entropy and thus reduce the free energetic advantage of a $2 \mathrm{D}$ coil compared to a 3D coil. Although we predominantly use Eq. (1) to describe the coil, we also consider the effects of assuming the coil is 3D.

At each step changes of configuration can only occur at the ends of the crystalline portion of the polymer. The possible processes are for the crystalline part of the polymer to grow by one unit by the extension of one of the stems or by the formation of a gauche bond as part of a fold, or for the crystalline part of the polymer to shrink by one unit. To preserve adjacent re-entry after the formation of the first of the two gauche bonds of a fold, the only possible moves are completion of the fold and removal of the first gauche bond. These possibilities are illustrated in Fig. 1. Generally we allow growth to occur from both ends of the crystalline configuration but, occasionally, in order to compare with theories of polymer crystallization, we only allow growth to occur from one end. Physically, these two scenarios correspond to the initial crystal nucleus being in the midsection of the polymer or at the end of the polymer, respectively. We see no reason why the latter should always be the case. The choice only makes a difference to the growth rate, which is of course twice as fast when growth can occur from both ends, and to the structure of the crystal near the initial nucleus.

Note that our scheme does not allow for the annealing of the part of the crystallite that has already been deposited. This will not affect our conclusions concerning the average thickness of the deposited layers but it may lead to an unrealistically rough appearance of the crystal. We will come back to this issue later in the article.

The change in the free energy of the polymer on taking a step, $\Delta A$, is given by $\Delta A=\Delta E_{\text {xtal }}+\Delta A_{\text {coil }}$, where $\Delta E_{\text {xtal }}$ is the change in energy of the crystalline configuration and $\Delta A_{\text {coil }}$ is calculated from Eq. (1) or (2). A rate is assigned to each of the possible moves. Each move is assumed to be an activated process with a barrier that is an energy $\Delta$ above the higher free energy state. The prefactor is assumed to be the same for all processes, and we set it to 1 thus defining a time unit. Therefore, the rate for a step is given by

$$
k_{i j}=\exp \left(\frac{-\Delta}{k T}\right) \min \left(1, \exp \left(\frac{-\Delta A_{i j}}{k T}\right)\right) .
$$

As the factor $\exp (-\Delta / k T)$ scales all the rates, simulations do not need to be carried out at different values of $\Delta$, but the results for one value can be scaled onto any other.

In the KMC simulation at each step we choose randomly one state, $j$, from those connected to the current state, $i$, with a probability given by

$$
P_{i j}=\frac{k_{i j}}{\sum_{j^{\prime}} k_{i j^{\prime}}},
$$

and update the time by an increment

$$
\Delta t=-\frac{\log (\rho)}{\sum_{j} k_{i j}},
$$

where $\rho$ is a random number in the range $[0,1]$. In this way the KMC algorithm simulates a stochastic process described by a Poisson distribution.

As the expressions for the various rates do not depend on the length of the polymer we do not set a limit to the length of the polymer, but allow each new crystalline layer to be formed from a single polymer.

Most of the results in this article are for the growth of a single crystalline layer on a growth face which is of uniform thickness. However, we have also considered the situation where many layers are grown on the initial crystal. In these simulations all except the first layer are grown on top of the previously grown layer and we only start to grow a new layer once the previous layer has been completed (when the crystalline polymer spans the periodic boundary conditions in the $a$ direction).

The thermodynamics of this model can be easily worked out if we ignore any entropic contribution to the crystal from variations in the stem length. The energy of a crystal with constant thickness, $l$, is

$$
E_{\mathrm{xtal}}=N_{\mathrm{xtal}}\left(\frac{2 \epsilon_{g}+\epsilon}{l}-2 \epsilon\right),
$$

where $N_{\text {xtal }}$ is the number of units in the crystalline configuration. The positive term is due to the surface energy of the fold surface. The minimum stable thickness, $l_{\min }$, can be calculated through the equation $E_{\text {xtal }} / N_{\text {xtal }}=A_{\text {coil }} / N_{\text {coil }}$. This gives

$$
l_{\min , 2 \mathrm{D}}=\frac{2 \epsilon_{g}+\epsilon}{\epsilon-k T \log \left(1+2 \exp \left(-\beta \epsilon_{g}\right)\right)}
$$

and

$$
l_{\min , 3 \mathrm{D}}=\frac{2 \epsilon_{g}+\epsilon}{2 \epsilon-k T \log \left(1+4 \exp \left(-\beta \epsilon_{g}\right)\right)} .
$$

Neglect of the entropy of the crystal means that these expressions are upper bounds. The temperature at which the surface 

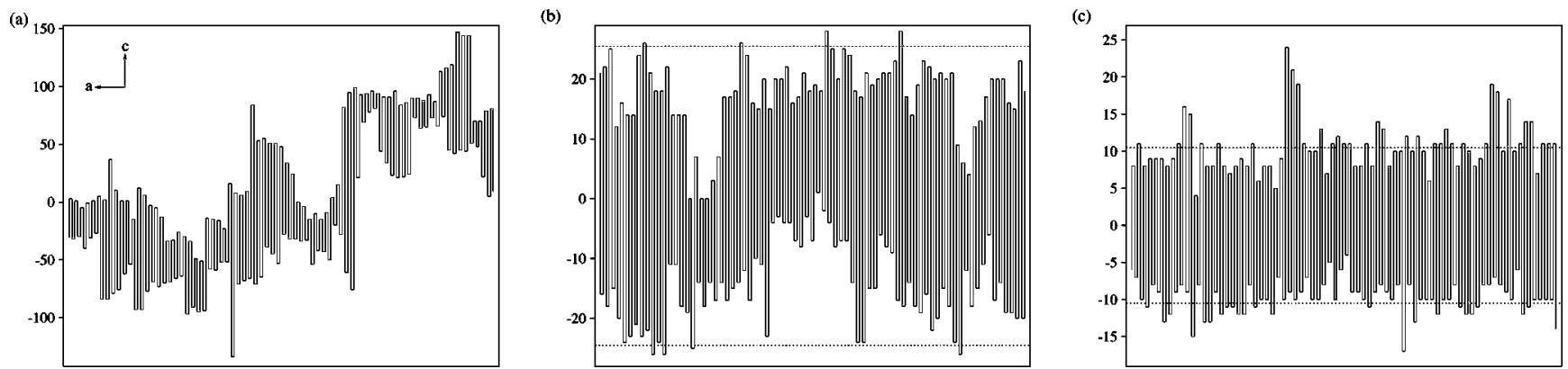

FIG. 2. Polymer configurations for a new crystalline layer grown at $T=2.75 \epsilon k^{-1}$ on surface thicknesses of: (a) $\infty$, (b) 50 and (c) 21 . Each configuration contains 150 stems and a dotted line marks the boundaries of the underlying surface; the $y$-axis origin is at the center of the surface.

of the polymer crystal loses its crystalline order, the "melting" or dissolution point, $T_{m}$, is given by the temperature for which the denominator is zero.

\section{RESULTS}

Some aspects of the typical behavior of our model for the growth of a single layer on a surface are illustrated in Figs. 2 and 3. Below the melting point $\left(T_{m}=4.06 \epsilon k^{-1}\right)$, for a sufficiently thick surface, a new crystalline layer grows. However, at low supercoolings the initial nucleation of a new layer can be quite slow. In the example shown in Fig. 3 (a), there is no net growth in the first million time steps, and only once a viable nucleus has formed does growth occur with relatively little impediment. This example clearly shows the effect on the dynamics of the free energy barrier for initial nucleation of a layer. The time scale for the initial nucleation event decreases with increasing supercooling and at large supercoolings it is not noticeable in the trajectories. (Very close to $T_{m}$ it is only feasible to grow new layers when a seed crystal is introduced.)

Above $T_{m}$, as one would expect, if we start the simulation with an initial seed crystal it duly dissolves. In the example shown in Fig. 3(b) the temperature is just above $T_{m}$, and so the rate of dissolution is slow because of the nearly flat free energy landscape. In every 1000 steps there are only three more dissolution than growth steps.

In the LH theory the thickness of a layer is determined by the length of the first stem that is deposited. However, as the free energy barrier associated with the first stem is proportional to the length of the stem, one could imagine that growth would be more rapid for a layer where the stem length gradually increases to its average value as crystallization progresses than for a layer with constant stem length. In such a configuration the lateral surface free energy is paid for "in installments" rather than all initially and growth is likely to be more rapid because of the lower initial free energy barrier.

Our model allows us to examine these questions easily. First, inspection of example configurations shows that there is considerable variation in the stem lengths within a layer (Fig. 2). There is nothing in the kinetics of growth which causes the stem length to be constant (although subsequent annealing of the new crystalline layer may reduce the variation in stem length). Second, we have tested whether there is any systematic dependence of the average stem length on the position of the stem with respect to the initial nucleation site (Fig. 4). Allowing the crystal to grow from only one end most closely mimics the conditions of the LH theory in which stems are grown one at a time. In this case we see that the suggestion we made above is confirmed. The length of the first stem is significantly shorter than the average stem length in the layer, and the stem length converges to the

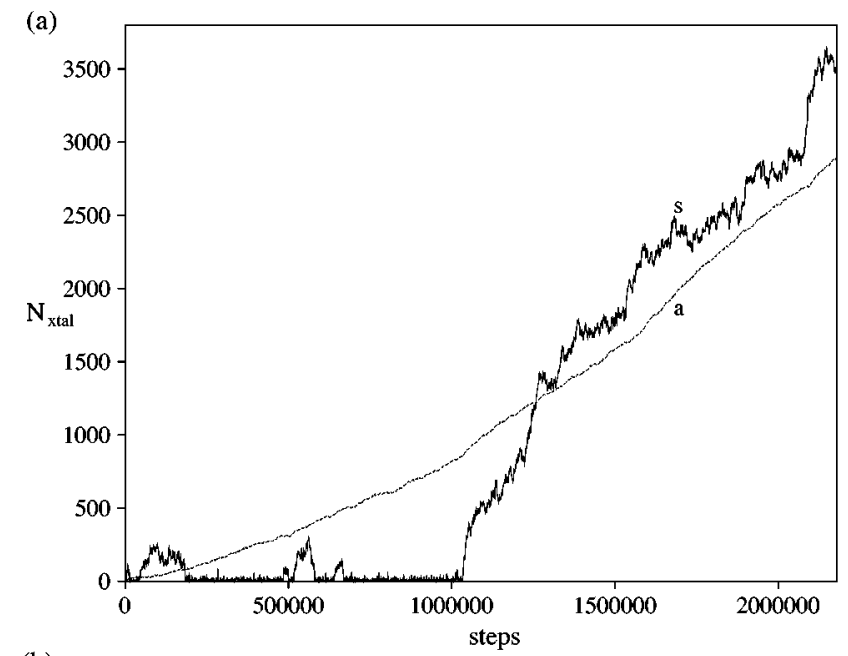

(b)

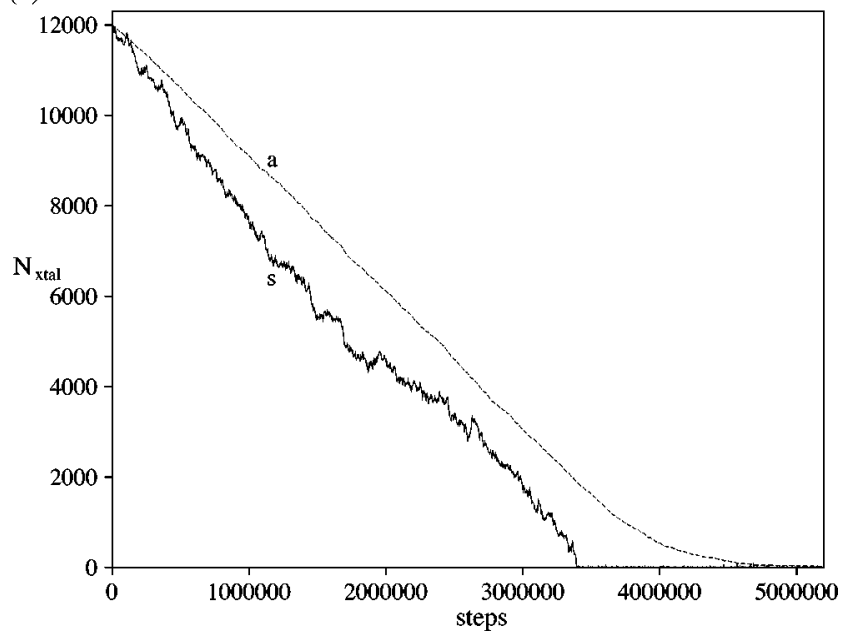

FIG. 3. The number of units in the crystalline layer, $N_{\text {xtal }}$, as a function of the number of steps for (a) $T=3.7 \epsilon k^{-1}$ and (b) $T=4.1 \epsilon k^{-1}$. The solid line (s) shows the behavior of a single layer, whereas the broken line (a) is an average over 50 layers. The underlying surface is infinite. Initially, there is in (a) a single unit on the surface and in (b) a crystal 100 stems wide, each 120 units long. 


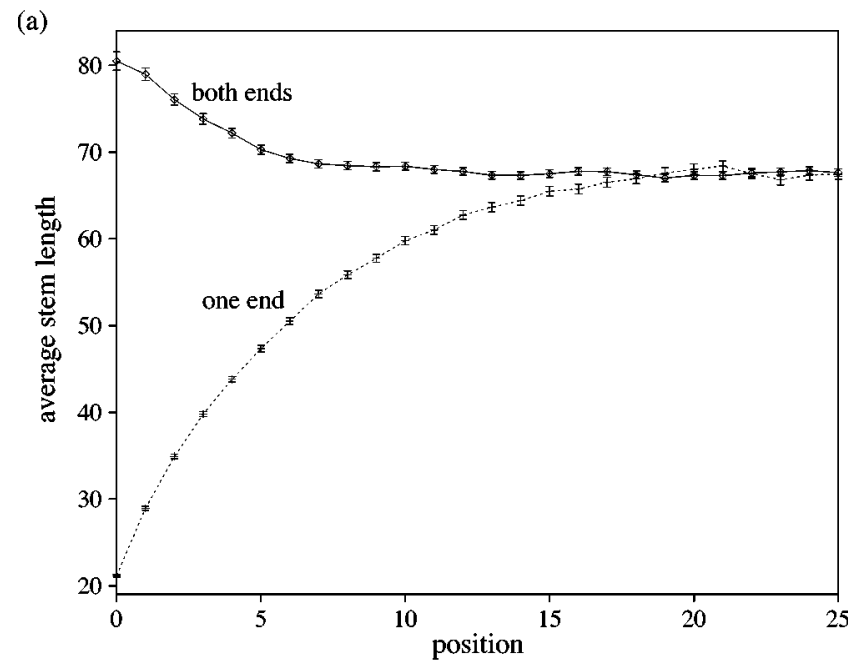

(b)

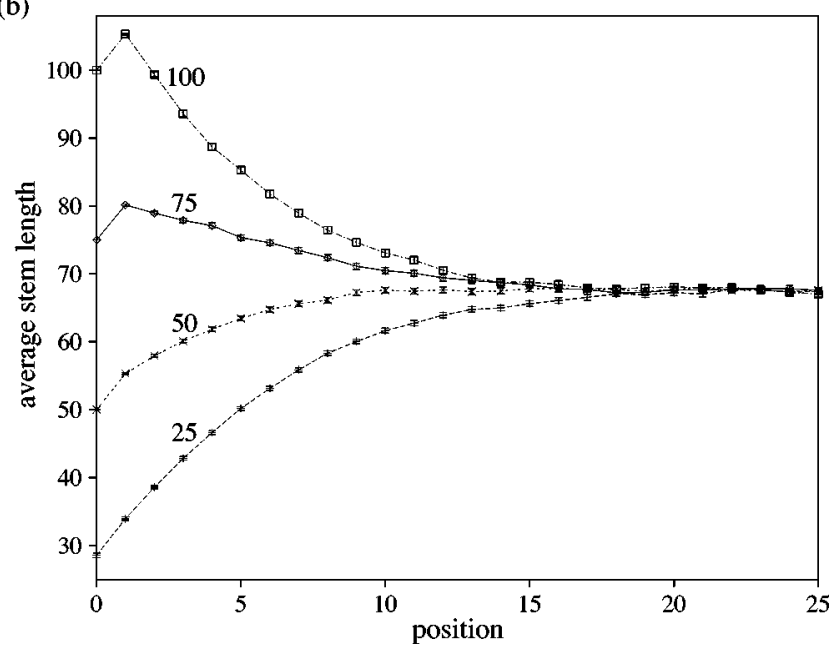

FIG. 4. The dependence of the average stem length on the distance of the stem from (a) the initial nucleation site and (b) the center of an initial crystal seed at $T=2.75 \epsilon k^{-1}$ for growth on an infinite surface. In (a) growth starts with a single polymer unit on the surface and we consider the cases where growth is allowed at one end or at both ends of the crystalline portion of the polymer. In (b) the crystal seeds are three stems wide; the lengths of the stems in the seeds are as labeled.

average value for the layer as the crystal grows.

Interestingly, when we allow growth to occur from both ends the initial behavior is very different [Fig. 4(a)]. This is because it is now possible to form an initial nucleus which consists of two stems connected by a fold which grow simultaneously, a scenario not considered in the LH theory. Such a nucleus is energetically favoured because of the interactions between the two stems. As it avoids the large free energy barrier associated with a single-stem nucleus ${ }^{37}$ there is now no advantage in the initial stems being shorter than the average in the layer [Fig. 4(a)]. The possibility of a two-stem nucleus was first suggested by Point ${ }^{24}$ and recently strong evidence for this nucleus has been obtained in a study of the free energy profiles along specific crystallization pathways. ${ }^{37}$

This finding about the structure of the initial nucleus puts a significant dent into LH theory because its assumption about the nature of the initial nucleus, and the resulting free energy barrier, is crucial to the theory. However, this finding is rendered somewhat irrelevant because the above results

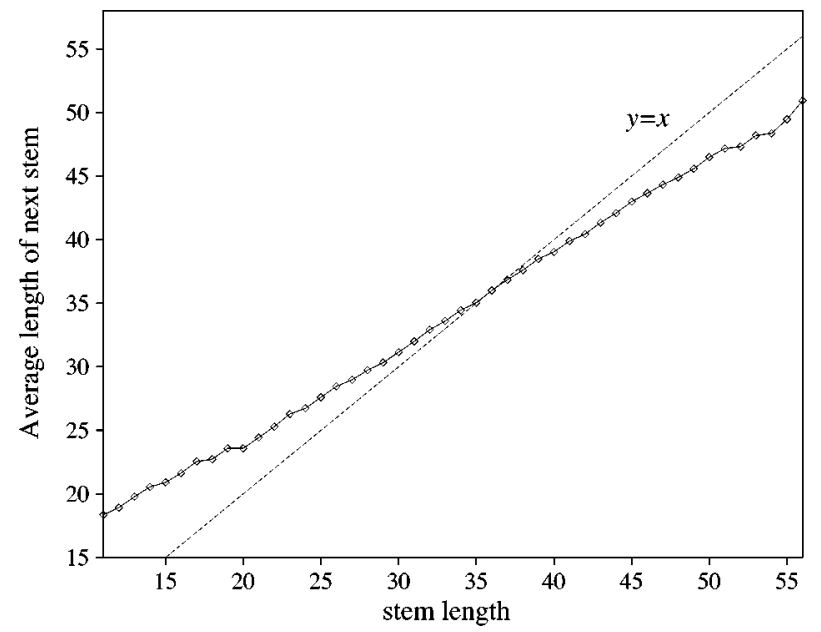

FIG. 5. The dependence of the stem length on the length of the previous stem for growth of a single layer on a surface 50 units thick at $T$ $=2.75 \epsilon k^{-1}$.

also undermine a more fundamental tenet of the LH theory, namely, the assumption that the thickness of a layer is determined by the initial nucleus. Further confirmation that this assumption does not hold comes when we examine the growth from initial seed crystals. Whatever the thickness of the initial seed the thickness of the growing crystal converges to the same value [Fig. 4(b)]. These results imply that the thickness of a crystalline layer must be determined by factors that are operating on the deposition of each stem and not those specific to the initial stems. We shall examine what these factors are in detail later.

In Fig. 5 we show the correlations between successively grown stems. Figure 5 has the form of a fixed-point attractor. It shows there is one stem length, $l^{*}$, for which the average length of the next stem is the same as the previous one (for Fig. $5 l^{*}=36$ ), and this corresponds to the average stem length in the layer. For stems longer (shorter) than $l^{*}$ the length of the next stem is on average shorter (longer) bringing the stem length nearer to $l^{*}$. Frank and Tosi have previously drawn a similar conclusion from their extension of the LH approach in which a single change in the stem length is allowed during the deposition of a new layer. ${ }^{13}$

In Fig. 6(a) we show how the thickness of a new layer depends on the temperature and the thickness of the growth surface. It can immediately be seen that the thickness of a new layer is not necessarily the same as the previous layer, thus contradicting another of the assumptions of the $\mathrm{LH}$ theory. Instead the temperature dependence of the curves reflects the factors that determine the thickness of a new layer. All the thickness curves rise as the temperature approaches $T_{m}$ because of the rise of $l_{\min }$; they end at the temperature where it is no longer possible to grow a new layer that is thermodynamically stable. [Figure 6(b) shows that the growth rate of the crystal goes to zero at these end points.] At low temperature the thickness also increases, in this instance, because it becomes increasingly difficult to scale the free energy barrier for forming a fold ${ }^{37}$ and so on average the stem continues to grow for a longer period. However, except for growth on an infinite surface, this rise is checked by the 
(a)

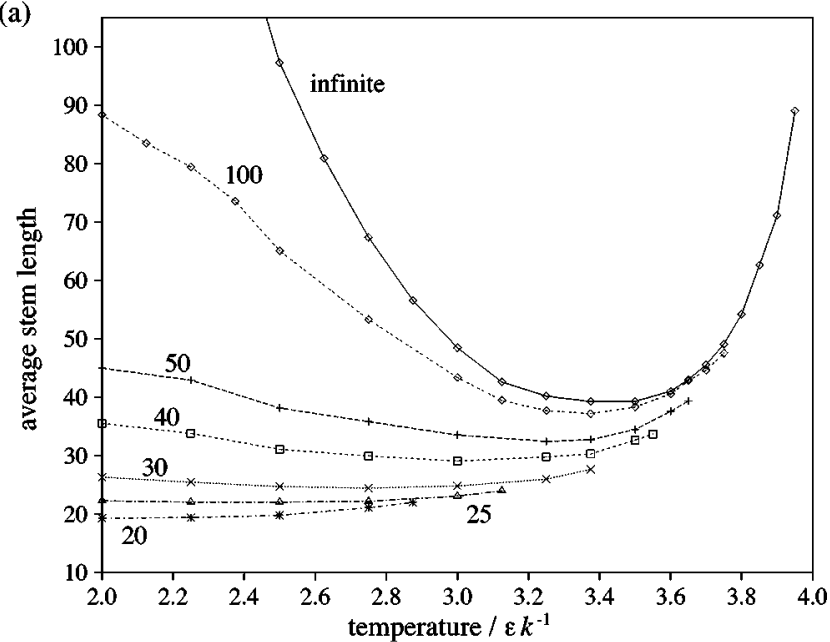

(b)

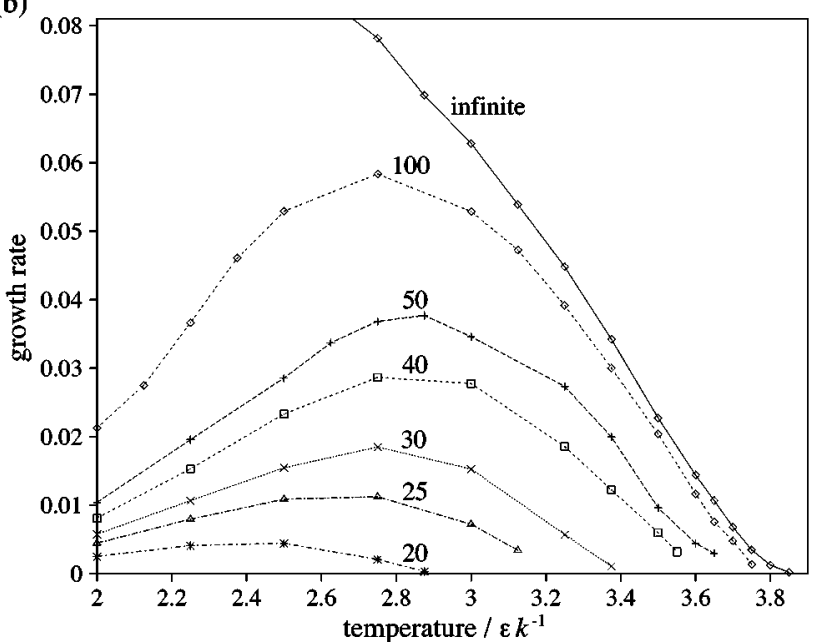

FIG. 6. (a) The average stem length and (b) the growth rate [polymer units per $\exp (-\Delta / k T)$ units of time] in the new crystalline layer as a function of temperature. The different curves are for different thicknesses of the underlying surface, as labeled.

presence of the edge of the underlying surface. It is unfavorable for the polymer to overhang the edge because these units do not gain the $-\epsilon$ energy of interaction with the surface.

An understanding of how the thickness of a polymer crystal is determined can be gained from these results but to do so it is better to plot the thickness of the new layer against the thickness of the growth surface at constant temperature, as in Fig. 7. In our KMC simulations we do not allow the crystalline layer to anneal. However, it is likely that some annealing does take place before the next layer is deposited. In what follows we consider two limits. The first is complete annealing. In that case we may replace the previous crystalline layer by a flat substrate with the same thickness. The second limit is no annealing. This we discuss with reference to Fig. 11. Let us first focus on the complete annealing limit. In that case we can use Fig. 7 to consider the effects of growing successive layers on top of each other. By following the dotted lines in Fig. 7 one sees what would happen for growth on a surface initially 50 units thick. For example, at $T=2.75 \mathrm{\epsilon k}^{-1}$ the first layer is 36 units thick, the second 28 , (a)

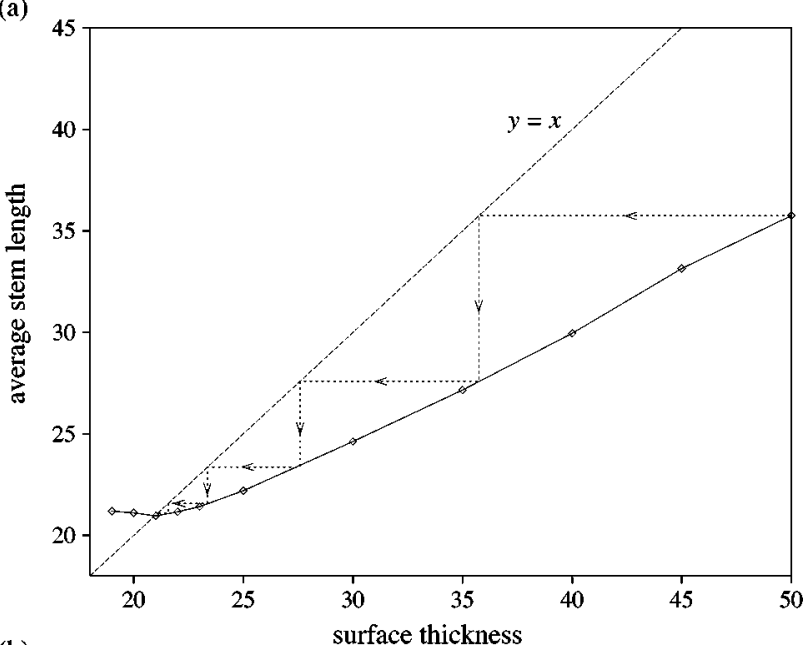

(b)

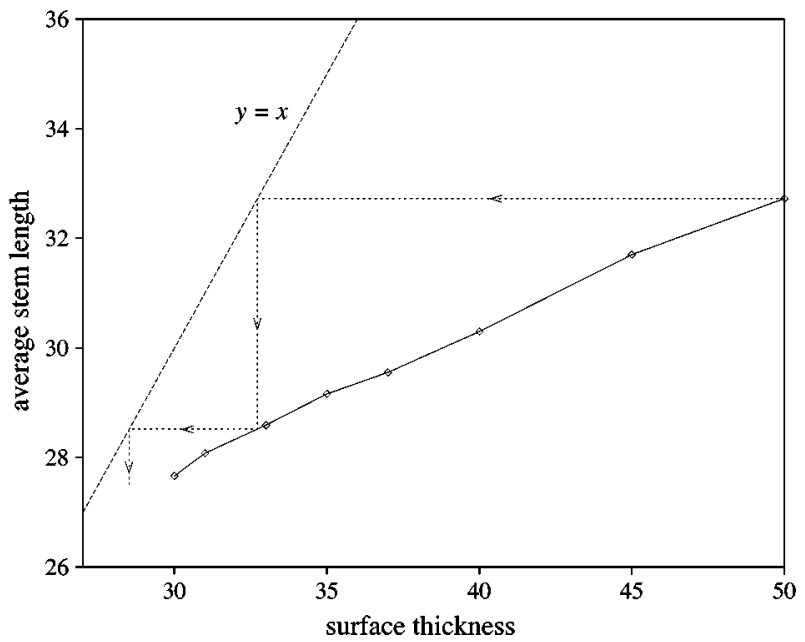

FIG. 7. The average stem length in the new crystalline layer as a function of the thickness of the underlying surface for (a) $T=2.75 \epsilon \mathrm{k}^{-1}$, and (b) $T$ $=3.375 \epsilon k^{-1}$. The dotted lines show how the thickness changes upon the addition of successive layers to a surface initially 50 unit thick.

the third $23, \ldots$ The thickness converges to the value $l^{* *}$ at which the curve crosses $y=x\left(l^{*} *=21\right.$ at $T$ $\left.=2.75 \epsilon k^{-1}\right)$, i.e., to the point where the thickness of the new layer is the same as the previous one, and then the crystal continues to grow at that thickness. Figure $7(\mathrm{a})$ is another example of a fixed-point attractor.

Again this picture is very different from that presented by the LH theory. It shows that it is inappropriate to compare the growth rates of crystals of different thickness because there is only one dynamically stable thickness, $l^{* *}$, for which growth at constant thickness can occur. For crystals which initially have a thickness different from $l^{* *}$, during growth the thickness will converge to $l^{* *}$, as has been observed in experiment. ${ }^{14,15}$ It is also interesting to note that the growth rate at $l^{* *}$ is not a maximum. Quite the contrary; growth of a thick crystal slows as the thickness decreases to $l^{* *}$ (Fig. 8).

Although this viewpoint contrasts with the LH theory, it was realized in a number of the early polymer crystallization papers ${ }^{13,22,39}$ that stable growth could only occur at the thickness for which a new layer has the same thickness as the previous one. However, since then this insight has for the 


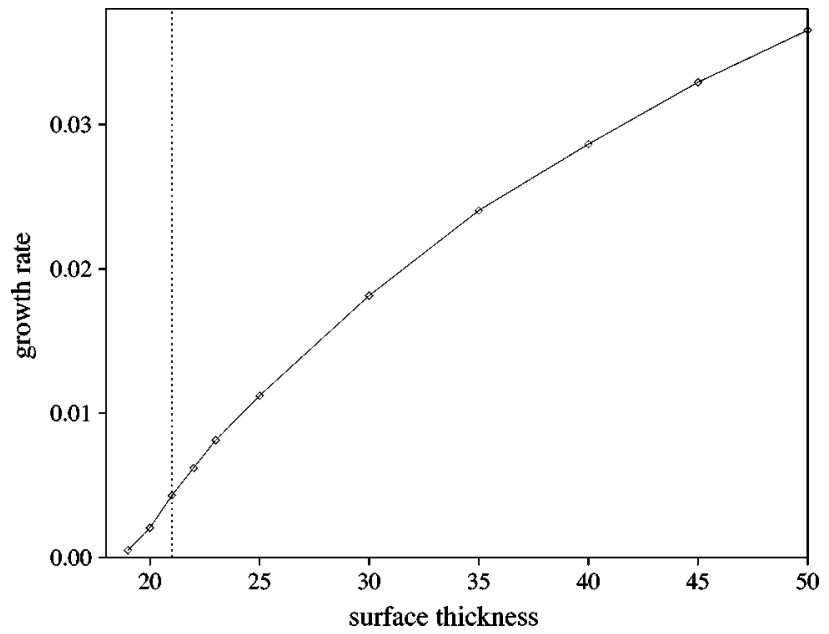

FIG. 8. The growth rate [polymer units per $\exp (-\Delta / k T)$ units of time] of the new layer as a function of the thickness of the underlying surface for $T$ $=2.75 \epsilon k^{-1}$. A dotted vertical line has been placed at thickness, $l^{* *}$.

most part been neglected, and, to the best of our knowledge, the iterative maps that underlie it have not been previously visualized. Also, in recent simulations by Chen and Higgs the lamellar thickness was observed to converge to the same value irrespective of the thickness of the initial seed crystal, ${ }^{28}$ but the significance of this behavior was not fully realized.

We can better understand the reasons for this behavior by examining representative polymer configurations (Fig. 2) and the probability distributions of the stem length (Fig. 9) for the growth of a single layer at a number of surface thicknesses. $l_{\text {min }}$ places one constraint on the stem length: only a small fraction of the stems can be shorter than $l_{\min }$ if the layer is to be thermodynamically stable. The boundary of the growth face places the second constraint on the stem length; it is energetically unfavorable for the polymer to extend beyond the edges of the underlying surface. Even in the absence of this constraint, i.e., on the infinite surface, the stem length remains finite because at every step there is always a finite probability that a fold will be formed, ${ }^{23,25}$ and so the probability distribution decays to zero at large stem lengths [Fig. 9(a)]. For the infinite surface any stem that is longer than $l_{\text {min }}$ is stable with respect to the coil and so a very wide range of stem lengths is observed (the standard deviation in the stem length is $\sim 35$ at $T=2.75 \epsilon k^{-1}$ ).

As can be clearly seen in Figs. 2 and 9, the thickness of the growth face exerts an increasingly strong influence on the new layer as the thickness decreases. For a surface 100 units thick the probability distribution of the stem length is very similar to the infinite case-again the maximum occurs at $\sim 40-50$ - except that the long tail of the distribution is cut off at 100 because the polymer rarely extends beyond the edge of the growth face. At this thickness the probability of the stem length being greater than the surface thickness is much less than it being smaller and, therefore, the new layer is significantly thinner than the underlying surface. As the surface thickness decreases the probability distributions of the stem length become increasingly narrow and the difference in probability between the stem length being greater or less than the surface thickness diminishes. This is because the range of viable stems - those with lengths larger than $l_{\min }$ but which do not significantly overhang the edge of the surface-become smaller. Finally, at $l^{* *}$ as the surface thickness approaches $l_{\min }$ the probability distribution become symmetrical about the surface thickness and the thickness of the new layer becomes equal to the thickness of the growth
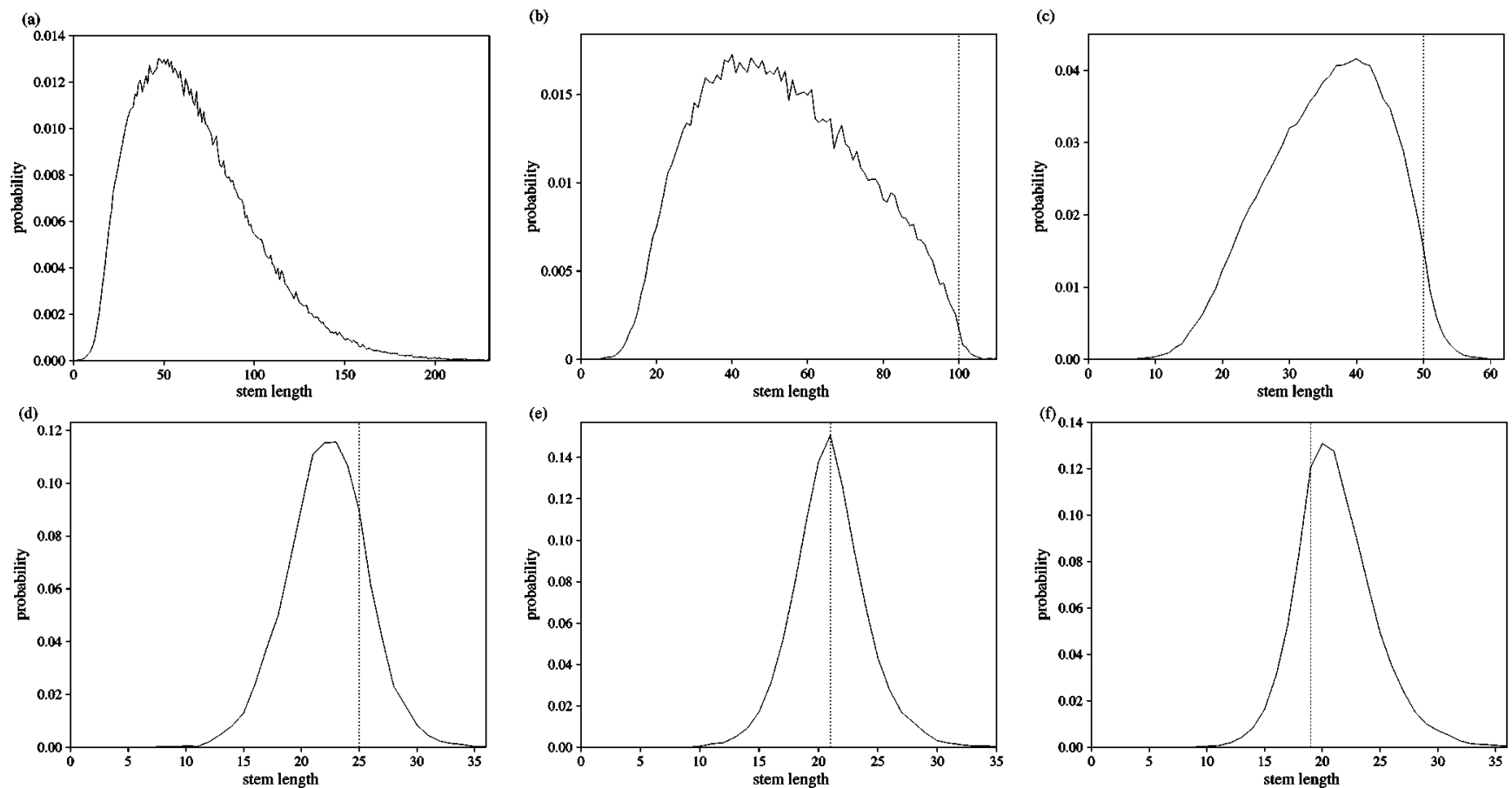

FIG. 9. Probability distributions of the stem length for a new crystalline layer grown at $T=2.75 \epsilon k^{-1}$ on surface thicknesses of: (a) $\infty$, (b) 100 , (c) 50 , (d) 25 , (e) 21 and (f) 19. The dotted vertical lines in the probability distributions are at the thickness of the underlying surface. 


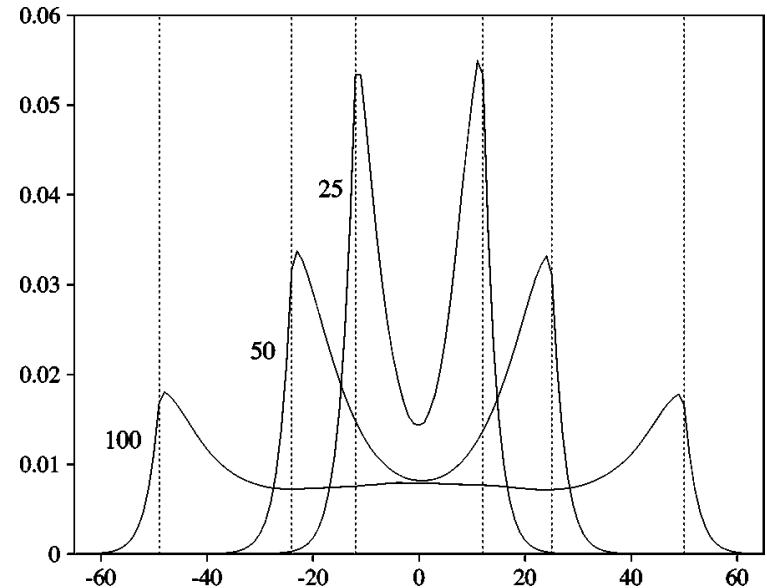

FIG. 10. The probability distribution for the position of the ends of the crystalline portion of the polymer for growth of a single layer on surfaces of different thicknesses, as labelled. The center of the surface is at 0 , and dotted lines are at the positions of the boundaries of the surface. $T$ $=2.75 \epsilon k^{-1}$.

surface [Fig. 9(e)]. For these reasons the thickness at which stable growth occurs is close to $l_{\min }$. For thicknesses less than $l^{* *}$ the asymmetry of the probability distribution is reversed [Fig. 9(f)].

It is also possible to understand why the dependence of the growth rate on thickness (Fig. 8) differs from the predictions of the LH theory. The cause is not the decrease of the thermodynamic driving force as the thickness decreases (this makes the growth rate go to zero at $l_{\min }$ ) since it is common to both this model and to LH theory. Rather, the difference arises mainly from the LH assumption that complete stems are deposited in a single step. Based upon comparisons of this approach with a fine-grained kinetic description of the deposition of a stem, Frank and Tosi showed that the LH complete-stem approach is reasonable if the only point on the fine-grained pathway at which a fold can occur is once a stem is complete. However, as has been pointed out previously, this condition does not hold for more realistic multipathway approaches. ${ }^{2}$ The fact that the polymer can form a fold at any point and can overhang the edge of the crystal in our model has a considerable effect on the growth rates. Such processes can lead to configurations that are not thermodynamically viable, such as when a new stem starts to form even though the previous one is less than $l_{\min }$ or when a stem significantly overhangs the edge of the crystal. These configurations have to be removed before growth can continue. The effect of such "blind alleys" is to reduce the growth rate. As the thickness of the surface decreases the range of stem lengths which give viable configurations decreases and so an increasing amount of time is spent searching blind alleys. These effects are reflected in the probability distributions for the position of the end of the crystalline polymer (Fig. 10). As the thickness decreases the end spends an increasing proportion of time near the edge of the growth surface waiting for the polymer to fold at a point when the stem has a length that is viable for further growth.

At $T=2.75 \epsilon k^{-1}$ there is no barrier to growth due to a rounded crystal profile. When the thickness is $l^{* *}$ the new layer has the same thickness as the previous layer. However, this scenario does not hold for all temperatures. At any temperature there is a thickness below which a new layer cannot grow because the surface is too thin [e.g., the last data point of the curve in Fig. 7(a) is for a surface 19 units thick], and there is no a priori reason why this must occur after the thickness curve has crossed $y=x$. Indeed, for $T>3.2 \epsilon k^{-1}$ there is no thickness for which successive layers have the same thickness. For example, at $T=3.375 \epsilon k^{-1}$ after the growth of two layers on a 50 unit thick surface the outer layer is $\sim 29$ units thick [Fig. 7(b)]; the crystal then stops growing because the outer layer is too thin. For these smaller supercoolings, as suggested in the entropic barrier model, the rounding of the crystal profile inhibits growth.

To overcome this barrier a cooperative mechanism is required whereby a new layer takes advantage of (and then locks in) dynamic fluctuations in the outer layer to larger thickness. The presence of such fluctuations is shown in the probability distributions of Fig. 9; however, growth in our model stops because we attempt to grow a new layer on an outer layer that is static. As overcoming the barrier would be most rapid when the magnitude of the fluctuations is the minimum necessary, we expect that this mechanism would lead to the crystal continuing to grow with the smallest thickness for which a new layer can grow, e.g., at $T$ $=3.375 \epsilon k^{-1}$ this is a thickness of 30 [Fig. 7(b)]. This mechanism again leads to a thickness for the polymer crystal which is close to $l_{\min }$.

It is interesting to note that behavior similar to our results has been found in some of the extensions of the LH theory which include multiple pathways in a restricted or approximate manner. In a typically prescient paper Frank and Tosi found that growth ceases at low supercoolings; ${ }^{13}$ however, without a mechanism to allow growth to continue, they concluded that polymer crystallization experiments could not correspond to this temperature range. Lauritzen and Passaglia were also aware that growth would cease in their model at low supercoolings, so they introduced an $\mathrm{ad}$ hoc energetic term into their rate constants that prevented this. $^{22,40}$

In using Fig. 7 to consider the growth of successive layers on the initial growth face we are assuming that all the roughness in the position of the fold surface is annealed out before a new layer starts to form. To check that the behavior we observe is not dependent on this assumption we have also performed simulations where we grow new layers directly on top of the previously grown layers. A typical cut through a crystal that was produced by this method is depicted in Fig. 11. We see the same mechanism of thickness determination as before: within 5-10 layers the thickness of the crystal converges to $l^{* *}$ and then growth continues at that thickness.

It is not clear which of these two limiting approaches is more realistic. In an atomic-force microscopy (AFM) study of the fold surface of polyethylene, only on a minority of the crystals could the folds be resolved clearly, ${ }^{41,42}$ suggesting that for most crystals the fold surface retains some roughness. However, the crystalline configurations generated by our model when the thickness of the growth face is much 


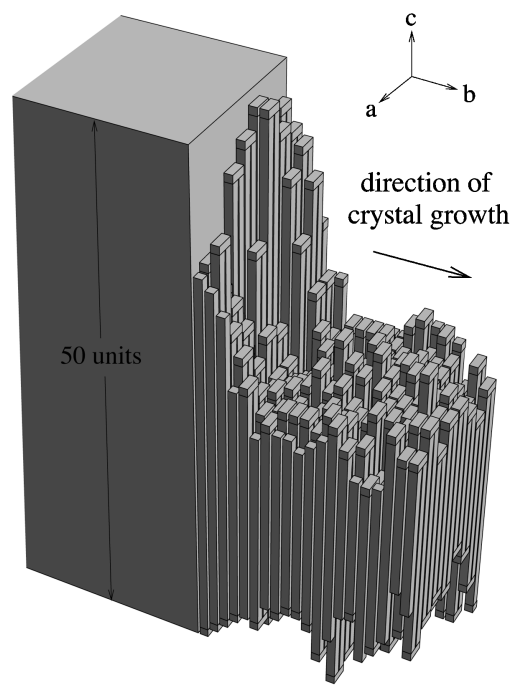

FIG. 11. Cut through a polymer crystal which was produced by the growth of 20 successive layers on a surface with a uniform thickness of 50 units at $T=2.0 \epsilon k^{-1}$. The stems are represented by vertical cuboids. The cut is 16 stems wide.

larger than $l_{\min }$ (Fig. 2) are clearly too rough. One would imagine that local rearrangements such as the one depicted in Fig. 12 would act to reduce the roughness while keeping the average stem length in the layer constant. More uniform configurations could have been obtained by including these mechanisms in our model but we did not do so because we would have to have set the relative rates of growth and annealing to an arbitrary value. To study the multilayer growth version of our model more systematically we would probably need to include these mechanisms because the roughness can at times hinder the growth; e.g., it can be hard for a new layer to grow across a section of the previous layer that involves a particularly short stem. Chen and Higgs experienced similar difficulties in their simulations. ${ }^{28}$

To determine the effect of our assumption that the disordered polymer is adsorbed onto the surface we have also performed simulations where the coil is assumed to be three dimensional. Figure 13(a) shows the dependence of the thickness of a new layer on the temperature and thickness of the surface. Figure 13(a) is similar in form to Fig. 6(a) except that the rise in the layer thickness at low temperature is not

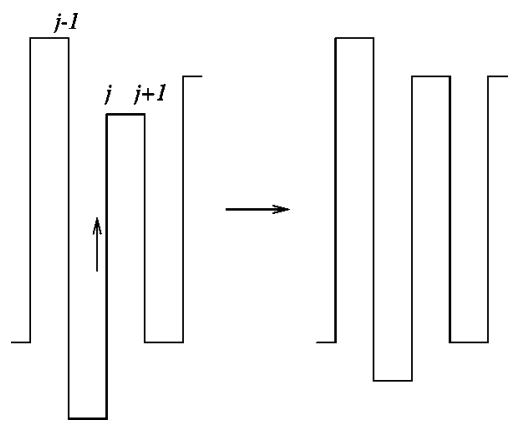

FIG. 12. An example of a mechanism by which the roughness in the crystalline layer could be annealed. The stem $j$ slides upwards decreasing the length of stem $j-1$ by 1 and increasing the length of stem $j+1$ by 1 . The change in energy for this rearrangement is $-\epsilon$.
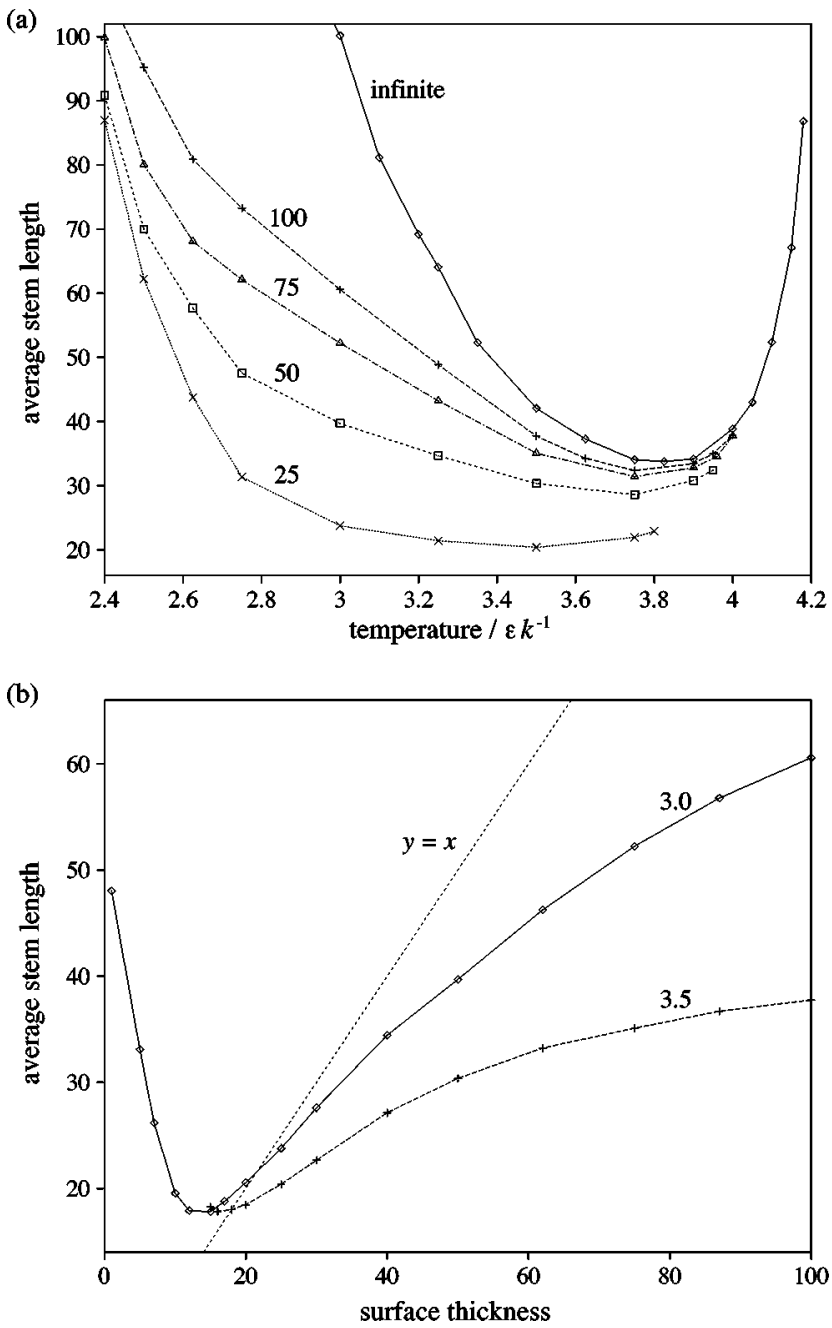

FIG. 13. The dependence of the average stem length in the new crystalline layer on the temperature and thickness of the surface when the disordered polymer is assumed to be an ideal three-dimensional coil. In (a) the values of the thickness of the underlying surface are shown, and in (b) the values of the temperature in $\epsilon k^{-1}$.

checked by the thickness of the underlying layer. The free energy difference between the coil and the crystal at low temperature is now large enough to allow the polymer to extend beyond the edge of the growth face. This has a corresponding effect on $l^{* *}$. When the coil was assumed to be two dimensional $l^{* *}$ was a monotonically increasing function of temperature which was always slightly larger than $l_{\min }$. However, now the value of $l^{* *}$ increases at low temperatures; e.g., at $T=3.0 \epsilon k^{-1} l^{* *}=21$ whereas at $T$ $=3.5 \epsilon k^{-1} \quad l^{* *}=18$ [Fig. 13(b)]. Assuming the coil to be three dimensional does not prevent the rounding of the crystal profile which causes growth to stop in our model at low supercoolings; however, the range of temperature for which this scenario holds is reduced $\left(T>3.8 \epsilon k^{-1}\right)$ because of the greater thermodynamic driving force for crystallization.

\section{CONCLUSIONS}

Our results present a new picture of the mechanisms that cause the thickness of lamellar polymer crystals to be constrained to a value just above $l_{\min }$. The free energetic costs 
of the polymer extending beyond the edges of the previous crystalline layer and of a stem being shorter than $l_{\text {min }}$ provide upper and lower constraints on the length of stems in a new layer. Their combined effect is to cause the crystal thickness to converge to a value close to $l_{\min }$ where growth with constant thickness then occurs.

This convergence of the thickness has been observed in experiments in which the thickness of growing polymer crystals adjusts to a change in temperature ${ }^{14,15}$ and in which lamellae form by epitaxial crystallization onto extendedchain crystalline fibers. ${ }^{43}$ It would be very interesting if AFM could be used to probe the profiles of the steps on the lamellae that result from temperature changes. From these profiles it would be possible to construct maps similar to Fig. 7(a) which could confirm the crucial role of the thickness of the growth face on the properties of a new crystalline layer. AFM could also be used to study the profile of the crystal close to the growth face in order to examine whether rounding of the crystal edge occurs.

Our results have significant implications for the LH surface nucleation theory. Although in some ways our model is in the LH tradition-new layers nucleate and grow by the addition of a succession of stems along the growth face - the removal of many of the LH constraints leads to a significantly different picture for the mechanism that determines the thickness of polymer crystals. Our work undermines many of the assumptions that are crucial to the LH theory: the initial nucleus is not a single stem; the initial nucleus does not determine the thickness within a layer; the thickness of a new layer is not necessarily the same as the previous layer; the observed thickness corresponds to the one value of the thickness, $l^{* *}$, for which crystals can grow with constant thickness and not to the thickness of those crystals which grow most rapidly in a fictitious ensemble of crystals of different thickness that grow with constant thickness; the growth rates are significantly affected by blind alleys (not included in the LH theory) which lead to configurations that are not viable for further growth. Of course, the attractive feature of the LH theory is that it provides analytical equations for the thickness and growth rate of polymer crystals that are in reasonable agreement with experiment. However, our results clearly indicate that the LH equations should be viewed as no more than phenomenological because their derivation relies upon an incorrect description of the physics of polymer crystallization.

The picture of polymer crystallization that comes from our results has more in common with the entropic barrier approach, and these two models are, in some ways, complementary. In the simulations of Sadler and Gilmer a realistic description of the polymer connectivity and folds was sacrificed in order to be able to study the full three dimensionality of the problem, whereas here we have a more realistic description of the polymer but could not at the same time include the cooperative interlayer dynamics that are necessary for growth at low supercoolings. Although Sadler and Gilmer's simulations have been interpreted in terms of the effects of a competition between an entropic barrier and a thermodynamic driving force on the growth rate, we expect that the mechanism of thickness determination is in fact similar to that for our model, namely, that during growth the crystal thickness converges to a thickness $l^{* *}{ }^{44}$ The cessation of growth that we see in our model at low supercoolings confirms that the rounding of the crystal profile does play a role in polymer crystallization. However, in our model this effect only occurs for a limited temperature range, whereas in the Sadler-Gilmer model rounding of the crystal profile occurs for all supercoolings.

We believe that the results in this article provide new insights into polymer crystallization, especially into the mechanism by which the thickness of a polymer crystal is attained. However, we stress that the model we use is simplified in order to make it computationally tractable. For example, we assume that nucleation only occurs once within a particular polymer; however, some simulations of a single polymer have seen crystalline domains develop within different parts of the polymer which at a later time coalesce to form a single crystal. ${ }^{27,29}$ Furthermore, we only allow growth processes to occur within the plane of the growth face and only within the outer layer; as explained earlier, the cessation of growth at low supercoolings is a result of these simplifications. This study, then, represents only a starting point in the application of computer simulation to understand polymer crystallization. It is hoped that simulation will lead to an increasingly refined picture of the microscopic mechanisms involved in the growth of polymer crystals.

\section{ACKNOWLEDGMENTS}

The work of the FOM Institute is part of the research program of Stichting Fundamenteel Onderzoek der Materie (FOM) and is supported by Nederlandse Organisatie voor Wetenschappelijk Onderzoek (NWO). One of the authors (J. P. K. D.) acknowledges the financial support provided by the Computational Materials Science program of the NWO. The authors thank Wim de Jeu for a critical reading of the manuscript.

\footnotetext{
${ }^{1}$ A. Keller, Philos. Mag. 2, 1171 (1957).

${ }^{2}$ For a balanced theoretical review, see, for example, K. Armistead and G. Goldbeck-Wood, Adv. Polym. Sci. 19, 219 (1992).

${ }^{3}$ A number of alternative theoretical approaches have also been proposed. For example, two new approaches to crystallization from the melt have been recently developed which make recourse to metastable phases: A. Keller, G. Goldbeck-Wood, and M. Hikosaka, Faraday Discuss. 95, 109 (1993); P. D. Olmsted, W. C. K. Poon, T. C. B. McLeish, N. J. Terrill, and A. J. Ryan, Phys. Rev. Lett. 81, 373 (1998).

${ }^{4}$ P. J. Barham, R. A. Chivers, A. Keller, J. Martinez-Salazar, and S. J. Organ, J. Mater. Sci. 20, 1625 (1985).

${ }^{5}$ J. I. Lauritzen and J. D. Hoffman, J. Res. Natl. Bur. Stand. 64, 73 (1960).

${ }^{6}$ J. D. Hoffman, G. T. Davis, and J. I. Lauritzen, in Treatise on Solid State Chemistry, edited by N. B. Hannay (Plenum, New York, 1976), Vol. 3, Chap. 7, p. 497.

${ }^{7}$ J. D. Hoffman and R. L. Miller, Polymer 38, 3151 (1997).

${ }^{8}$ D. M. Sadler and G. H. Gilmer, Polymer 25, 1446 (1984).

${ }^{9}$ M. A. Spinner, R. W. Watkins, and G. Goldbeck-Wood, J. Chem. Soc., Faraday Trans. 91, 2587 (1995).

${ }^{10}$ D. M. Sadler and G. H. Gilmer, Phys. Rev. Lett. 56, 2708 (1986)

${ }^{11}$ D. M. Sadler, Nature (London) 326, 174 (1987).

${ }^{12}$ D. M. Sadler and G. H. Gilmer, Phys. Rev. B 38, 5684 (1988).

${ }^{13}$ F. C. Frank and M. Tosi, Proc. R. Soc. London, Ser. A 263, 323 (1961).

${ }^{14}$ D. C. Bassett and A. Keller, Philos. Mag. 7, 1553 (1962).

${ }^{15}$ M. Dosière, M.-C. Colet, and J. J. Point, J. Polym. Sci., Polym. Phys. Ed. 24, 345 (1986).

${ }^{16}$ D. M. Sadler, Polymer 24, 1401 (1983).
} 
${ }^{17}$ D. M. Sadler and G. H. Gilmer, Polym. Commun. 25, 196 (1984).

${ }^{18}$ D. M. Sadler, Polym. Commun. 27, 140 (1986).

${ }^{19}$ D. M. Sadler, J. Chem. Phys. 87, 1771 (1987).

${ }^{20}$ D. M. Sadler, Polymer 28, 1440 (1987).

${ }^{21}$ The probability distribution of the stem length in the outermost layer has been taken as providing evidence of a free energy barrier (Refs. 8 and 12).

However, this probability distribution is a steady-state distribution under conditions of growth, whereas the free energy can only be related to equilibrium probability distributions.

${ }^{22}$ J. I. Lauritzen and E. Passaglia, J. Res. Natl. Bur. Stand. 71, 261 (1967).

${ }^{23}$ J. J. Point, Macromolecules 12, 770 (1979).

${ }^{24}$ J.-J. Point, Faraday Discuss. Chem. Soc. 68, 167 (1979).

${ }^{25}$ E. A. DiMarzio and C. M. Guttman, J. Appl. Phys. 53, 6581 (1982).

${ }^{26}$ J.-J. Point, Faraday Discuss. Chem. Soc. 68, 366 (1979).

${ }^{27}$ T. Yamamoto, J. Chem. Phys. 107, 2653 (1997).

${ }^{28}$ C.-M. Chen and P. G. Higgs, J. Chem. Phys. 108, 4305 (1998).

${ }^{29}$ L. Toma, S. Toma, and J. A. Subirana, Macromolecules 31, 2328 (1998).

${ }^{30}$ C. Liu and M. Muthukumar, J. Chem. Phys. 109, 2536 (1998).

${ }^{31}$ T. Yamamoto, J. Chem. Phys. 109, 4638 (1998).

${ }^{32}$ J. P. K. Doye and D. Frenkel, Phys. Rev. Lett. 81, 2160 (1998).

${ }^{33}$ The main effect of $\epsilon_{g}$ is through its effect on $l_{\text {min }}$ [Eqs. (7) and (8)].
${ }^{34}$ S. Doniach, T. Garel, and H. Orland, J. Chem. Phys. 105, 1601 (1996).

${ }^{35}$ U. Bastolla and P. Grassberger, J. Stat. Phys. 89, 1061 (1997).

${ }^{36}$ J. P. K. Doye, R. P. Sear, and D. Frenkel, J. Chem. Phys. 108, 2134 (1998).

${ }^{37}$ J. P. K. Doye and D. Frenkel, J. Chem. Phys. 109, 10033 (1998).

${ }^{38}$ A. F. Voter, Phys. Rev. B 34, 6819 (1986).

${ }^{39}$ F. P. Price, J. Chem. Phys. 35, 1884 (1961).

${ }^{40}$ Lauritzen and Passaglia introduced an energetic penalty if a stem length, $l$, differed from the thickness of the surface, $l_{s}$, which was proportional to $\left|l-l_{s}\right|$. If $l>l_{s}$ this is reasonable since the polymer no longer interacts with the surface. However, there is no physical basis for this term when $l<l_{s}$; it just artificially constrains $l$ to have a value similar to $l_{s}$.

${ }^{41}$ R. Patil and D. H. Reneker, Polymer 35, 1909 (1994).

${ }^{42}$ S. N. Magonov and D. H. Reneker, Annu. Rev. Mater. Sci. 27, 175 (1997).

${ }^{43}$ A. Keller, Faraday Discuss. 68, 145 (1979).

${ }^{44}$ This has been confirmed in recent simulations of the Sadler-Gilmer model [J. P. K. Doye and D. Frenkel, J. Chem. Phys. (submitted) (cond-mat/ 9812088)]. The effect is also implicit in some of the results of Ref. 45.

${ }^{45}$ G. Goldbeck-Wood, Polymer 31, 586 (1990). 